\title{
TRAUMA ABDOMINAL: ESTUDO DAS LESÕES MAIS FREQUENTES DO SISTEMA DIGESTÓRIO E SUAS CAUSAS
}

\author{
Abdominal trauma: study of the most frequent wounds of digestive system and its \\ causes
}

\author{
Jurandir Marcondes RIBAS-FILHO, Osvaldo MALAFAIA, Marcelo Morikuni FOUANI, Marcel da Silva JUSTEN, \\ Lucas Eduardo PEDRI, Letícia Mayer Alves da SILVA, João Felippe MENDES
}

ABCDDV/619

Ribas-Filho JM, Malafaia O, Fouani MM, Justen MS, Pedri LE, Silva LMA, Mendes JF. Trauma abdominal: estudo das lesões mais frequentes do sistema digestório e suas causas. ABCD Arq Bras Cir Dig 2008;21(4): 170-4

RESUMO - Racional - O trauma abdominal é o sofrimento resultante de uma ação súbita e violenta por diversos agentes. Sua incidência vem aumentando e a gravidade é determinada pela lesão de estruturas vitais do abdome e pela associação com outras lesões. Objetivo - Identificar as causas do trauma abdominal, relacionar as vísceras digestivas mais atingidas, a existência de lesões em outras regiões e as suas relações com sexo e faixa etária. Método - Foram selecionados 34 pacientes do Sistema Único de Saúde com diagnóstico de trauma abdominal, atendidos no período de janeiro de 2005 a setembro de 2005 no Hospital Universitário Evangélico de Curitiba. A coleta dos dados foi realizada com o auxílio de um protocolo previamente elaborado. Resultados - Constatou-se que $91 \%$ das vítimas eram do sexo masculino. A faixa etária mais atingida foi a terceira década. Quanto à classificação dos traumatismos, $58,82 \%$ apresentaram ferimentos abertos e $41,18 \%$ contusões. As quedas foram responsáveis por $44 \%$ das contusões, seguidas por acidentes automobilísticos com $35 \%$. As origens mais comuns dos ferimentos abertos foram aquelas provocadas por armas de fogo em $56 \%$ e as por armas brancas em $44 \%$ dos casos. Ocorreu lesão no intestino delgado em $31 \%$ dos ferimentos abertos, seguido de lesão hepática, cólon e rim, cada uma com $23 \%$. Nas contusões, $60 \%$ dos pacientes sofreram ferimentos esplênicos. O tórax foi a região mais associada ao trauma abdominal (31\%). Conclusão - As principais causas de trauma abdominal foram ferimentos por arma de fogo (trauma aberto) e quedas (trauma fechado). As vísceras mais atingidas foram as parenquimatosas (baço e fígado) no trauma fechado e lesão de intestinos, fígado e rins no trauma aberto. A maioria dos pacientes era do sexo masculino com predomínio da terceira década.

DESCRITORES - Traumatismos Abdominais. Contusões. Ferimentos e Lesões. Armas de fogo. Acidentes de trânsito.

\section{INTRODUÇÃO}

O trauma abdominal é o sofrimento resultante de uma ação súbita e violenta, exercida contra o abdome por diversos agentes causadores: mecânicos, químicos, elétricos e irradiações. A incidência desse traumatismo vem aumentando progressivamente e sua gravidade é determinada pela lesão de órgãos ou estruturas vitais do abdome e pela associação com outras lesões, principalmente crânio e tórax ${ }^{16}$.

O sucesso no manejo do trauma abdominal é caracterizado pela eficiência da abordagem inicial que permite instituir o diagnóstico precoce e o tratamento oportuno das lesões intra-abdominais, quando presentes.

Classifica-se esse trauma em dois tipos principais - aberto ou fechado. No aberto existe solução de continuidade da pele; enquanto que no fechado, também denominado contusão abdominal, a pele está íntegra, sendo que os

Trabalho realizado no Programa de Pós-Graduação em Princípios da Cirurgia do Hospital Universitário Evangélico de Curitiba/ Faculdade Evangélica do Paraná. Curitiba, PR, Brasil.

Correspondência: Jurandir Marcondes Ribas Filho, e-mail: ipem@evangelico.org.br efeitos do agente agressor são transmitidos às vísceras através da parede abdominal, ou se dão por contragolpe ou desaceleração. Por sua vez, os traumatismos abertos são subdivididos em penetrantes e não penetrantes na cavidade abdominal.

Os ferimentos abdominais abertos são usualmente causados por armas de fogo ou por armas brancas ${ }^{19}$.

As causas mais frequentes da contusão são os acidentes automobilísticos $(70 \%)$, os golpes $(17 \%)$ e as quedas acidentais (6\%). As contusões são responsáveis por $1 \%$ de todas as internações hospitalares por trauma abdomina ${ }^{17}$.

Nas crianças, além dos mecanismos de lesão previamente mencionados, devem também ser considerados o abuso físico e o trauma secundário à atividades recreativas, tais como ciclismo, natação e skating ${ }^{19}$.

Com o abdome traumatizado podem ocorrer lesões nos diversos órgãos e estruturas intra-abdominais, levando a ruptura de vísceras ocas e/ou parenquimatosas. As vísceras parenquimatosas lesadas ocasionam perda sanguínea, podendo levar à hemorragias importantes, enquanto que as vísceras ocas lesadas causam liberação de secreções digestivas como suco gástrico ou intestinal, bile, fezes e 
urina, podendo levar à peritonite.

Nas contusões, lesam-se os órgãos com maior teor de água, menor resistência da cápsula e mais fixos ${ }^{13}$.

As lesões viscerais estão presentes em até $98 \%$ dos casos de ferimentos abertos por arma de fogo ${ }^{9}$. Os órgãos mais atingidos nesse trauma do tipo aberto são aqueles que ocupam maior área, como por exemplo, o fígado ${ }^{13}$.

No atendimento inicial aos pacientes com trauma abdominal é de grande importância o estabelecimento de prioridades. Em primeiro momento, a manutenção da via aérea pérvia, com controle da coluna cervical, além da avaliação da respiração e controle da hemorragia são essenciais e determinantes da sobrevida destes pacientes.

Quando o trauma abdominal não é isolado, havendo lesões em outras regiões, o atendimento inicial envolve o $\mathrm{ABCDE}$ do politraumatizado. Assim, uma série de prioridades e princípios devem ser seguidos envolvendo terapêutica e equipe multidisciplinar. $\mathrm{O}$ atendimento é diretamente proporcional à gravidade do trauma, sendo que as avaliações laboratoriais e radiológicas dependem das condições e necessidades de cada doente e, muitas vezes, a laparotomia se impõe de imediato 16.

O objetivo desse estudo é identificar as causas do trauma abdominal, relacionar com as vísceras do sistema digestório mais atingidas, a existência de lesões associadas em outras regiões e as suas relações com o sexo e faixa etária.

\section{MÉTODO}

O estudo foi realizado no Hospital Universitário Evangélico de Curitiba (HUEC) e aprovado pelo Comitê de Ética e Pesquisa da Faculdade Evangélica do Paraná (FEPAR). Foi elaborado a partir de 34 prontuários de pacientes do Sistema Único de Saúde (SUS), atendidos no período de janeiro de 2005 a setembro de 2005.

A coleta de dados deu-se a partir de prontuários, de forma retrospectiva, por meio de protocolo elaborado previamente, baseado nas variáveis que constituem os objetivos principais deste trabalho (Figura 1).

Para facilitar o estudo, os traumas abdominais foram divididos em: trauma aberto (ferimentos por armas de fogo ou por arma branca) e trauma fechado (contusão). Dentro de cada divisão foram analisadas as vísceras e as regiões associadas mais atingidas.

\section{RESULTADOS}

Constatou-se que a maioria das vítimas era do sexo masculino (91\%). A faixa etária dos pacientes variou de 11 a 70 anos, sendo que o maior número de casos ficou entre 21 a 30 anos (Gráfico 1).

Analisando a causa do trauma, observou-se que predominou o trauma abdominal do tipo aberto com 20 casos analisados, sendo que o fechado de 14 casos (Gráfico 2).

Dentre os traumas abertos, as principais causas foram os ferimentos por arma de fogo (Gráfico 3).

As principais causas das contusões foram as quedas, representando $44 \%$ dos casos, seguido de acidentes automobilísticos (35\%) (Gráfico 4).

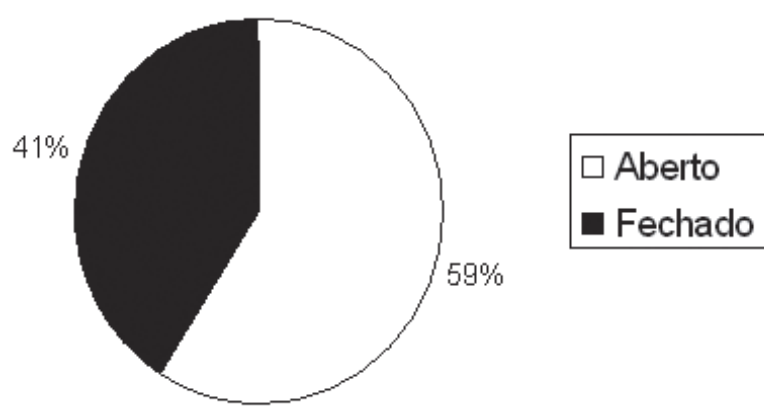

GRÁFICO 1 - Relação entre sexo e idade no trauma abdominal

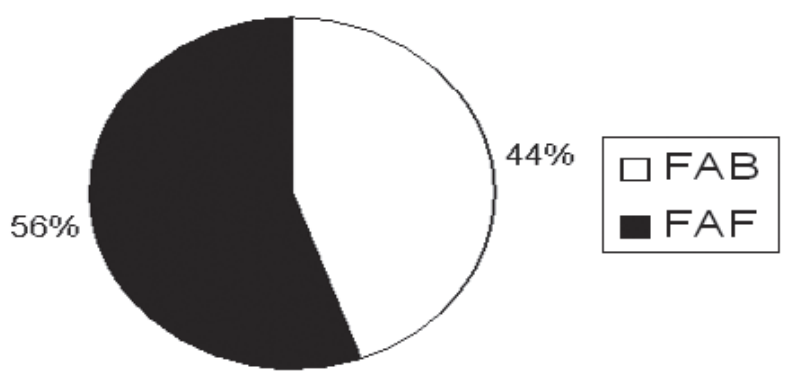

GRÁFICO 2 - Tipos de trauma

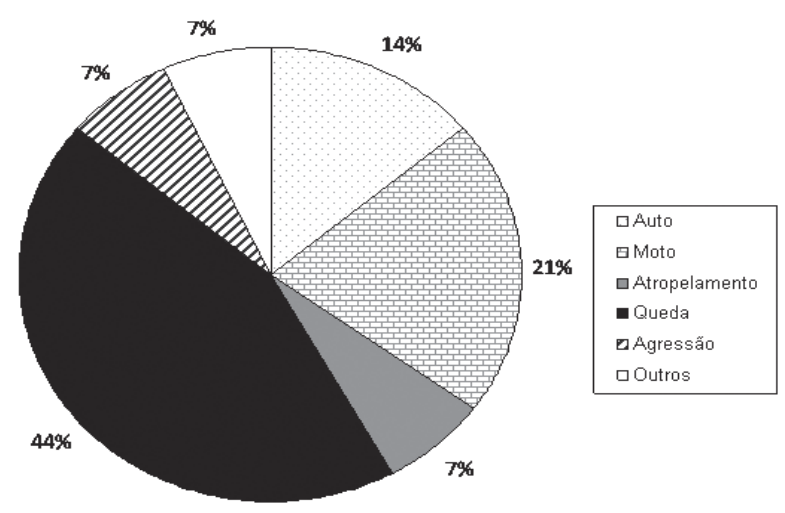

GRÁFICO 3 - Causas de trauma abdominal aberto

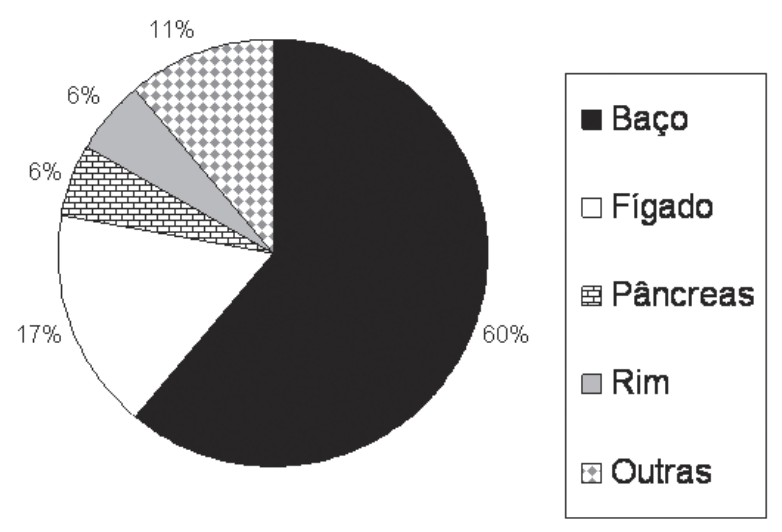

GRÁFICO 4 - Causas de trauma abdominal fechado 
Na análise das lesões por contusões, as vísceras mais atingidas foram respectivamente: baço e fígado, sendo pouco comuns as lesões pancreáticas e renais (Gráfico 5).

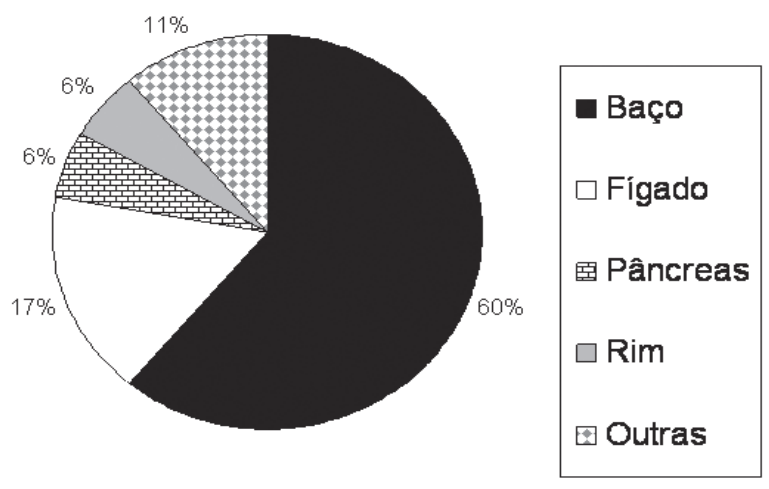

GRÁFICO5-Vísceras mais atingidas em traumas abdominais fechados

As vísceras mais atingidas no trauma abdominal aberto foram o intestino delgado, seguido de fígado, rins e cólon (Gráfico 6).

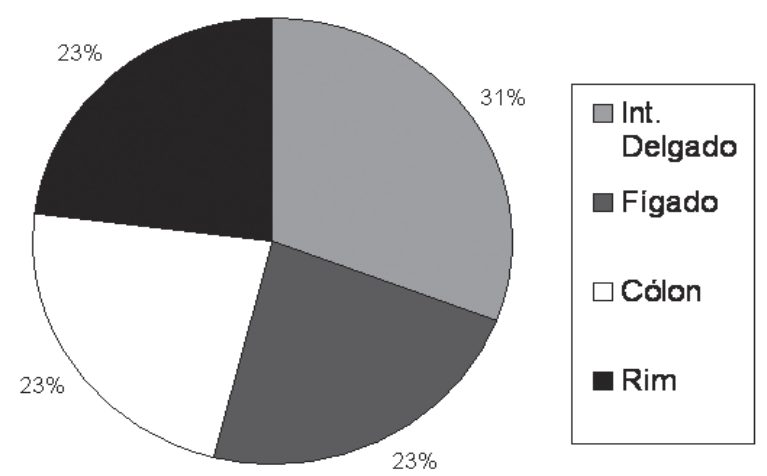

GRÁFICO 6 - Vísceras mais atingidas em traumas abdominais abertos

Observou-se que os pacientes com trauma abdominal, também podem apresentar lesões associadas em outras regiões, caracterizando um politraumatizado $(69,23 \%)$. Sendo assim, o tórax foi a principal região associada e o crânio a área menos atingida (Gráfico 7).
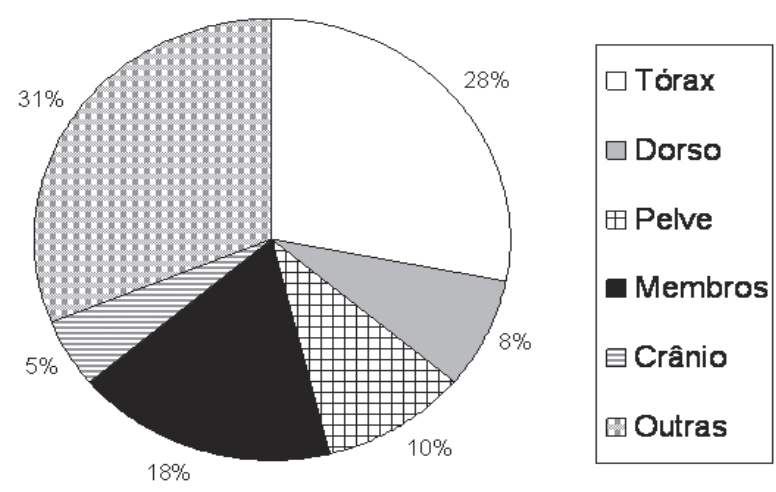

GRÁFICO 7 - Outras regiões atingidas no trauma abdominal

\section{DISCUSSÃO}

O número de pacientes traumatizados tem aumentado nos últimos anos e, grande parte desses, se deve ao aumento da violência ${ }^{5}$ e da energia cinética envolvida nos acidentes de trânsito, principalmente nas médias e grandes cidades $^{21}$. Embora nos últimos quatro anos tenha havido sensível redução nos índices de mortalidade por acidentes de trânsito nas principais capitais brasileiras, esse ainda se mantém como importante causa de morbimortalidade ${ }^{1}$. Nas Américas, os acidentes com automotores são as principais causas externas de mortalidade ${ }^{4,20}$. Isso causa aumento da complexidade das lesões encontradas nas vítimas e, consequentemente, em um novo desafio para os cirurgiões.

Quando há instabilidade hemodinâmica, sinais de peritonite, lesões penetrantes ou distensão abdominal, o diagnóstico pode ser feito mais rapidamente, porém algumas vezes são necessários exames complementares. O FAST (Focused Assesment With Sonography In trauma) pode ser usado devido à sua alta sensibilidade em detectar hemoperitônio em pacientes instáveis e o lavado peritoneal é útil quando o ultra-som e a tomografia não estão disponíveis. Já quando o paciente encontra-se estável, o método de escolha, em pacientes com trauma abdominal contuso, é a tomografia computadorizada com triplo contraste $^{22}$.

Pelos resultados obtidos nessa pesquisa, houve predominância do sexo masculino (91\%), conforme outros trabalhos encontrados na literatura ${ }^{14,15,17,18,22}$ e semelhante ao relato do Colégio Americano de Cirurgiões, com $64,6 \%{ }^{11}$.

Nessa casuística, a faixa de idade mais atingida foi a terceira década de vida, o que se assemelha com outros estudos $^{17,21}$. Portela ${ }^{14}$ cita que essa tendência deve-se a alguns fatores tais como pertencer à faixa etária mais produtiva da vida, praticar esportes com maior frequência, estar vinculado à atividades de combate e o maior consumo de bebidas alcoólicas. Existem alguns autores que apontam a quarta década como a de maior frequência ${ }^{12}$.

Assim como no trabalho de Stalhschmidt ${ }^{21}$ e Prado Filho ${ }^{15}$ a presente pesquisa como a maioria dos traumas o do tipo aberto, ao contrário do predomínio de contusões referidas em Ribas Filho ${ }^{17}$ e Portela ${ }^{14}$. Armas de fogo foram os instrumentos mais utilizados para causar os ferimentos abertos, o que são corroborados por outros autores ${ }^{17,19}$. Entretanto esse dado discorda de outros que citam como instrumento mais usado as armas brancas ${ }^{15}$.

As quedas foram as principais responsáveis pelos traumas abdominais fechados, igualmente referido no trabalho de Batista3, porém diferente da posição deste evento traumático na escala de prevalência do Brasil $\left(5^{\circ}\right.$ lugar) ${ }^{23}$ e diferente dos trabalhos onde prevaleceram os acidentes de trânsito ${ }^{7,15,17}$. No idoso a queda constitui causa bastante frequente de traumatismo, porém a população observada neste estudo não condiz com essa.

O baço foi a principal víscera comprometida nas contusões, corroborando com outras publicações ${ }^{7,8,11,14,17}$. 
Outra víscera bastante acometida nessas contusões é o fígado - as lesões leves correspondem à maioria (85\%) das lesões hepáticas ${ }^{6}$.

Em traumas abertos as vísceras mais atingidas foram intestino delgado, seguido de fígado, rins e cólon, possuindo certa equivalência com os resultados de outros estu$\operatorname{dos}^{16,17,21,26}$. Neste estudo, o fígado foi o segundo órgão mais envolvido nos ferimentos abertos, justificando a afirmação de Fagundes ${ }^{10}$ sobre avaliar as lesões hepáticas, visto que é o órgão sólido mais lesado nos ferimentos abdominais por arma de fogo, com mortalidade geral de $17 \%$.

Lesões associadas em outras regiões foram encontradas em $69,23 \%$ dos pacientes. O alto número de lesões associadas é importante desafio para a condução dos pacientes, pois sua presença dificulta a decisão do tratamento conservador. Na amostra utilizada a região mais afetada foi o tórax, igualmente em trabalho de Ribas Filho ${ }^{17}$.

O trauma, muitas vezes é considerado a "doença negligenciada da sociedade moderna" porque mata e incapacita mais do que as guerras e, mesmo assim, não sensibiliza nem mobiliza a sociedade e os governos. $\mathrm{O}$ prejuízo social que o trauma traz é imensurável. Não há como avaliar quantitativamente o impacto social que as mortes ou sequelas do trauma causam para a população. A etiologia do trauma está na sociedade e nela a solução. Cada ação política, preventiva, terapêutica e de consciência debruçada sobre a pessoa humana é uma esperança para atenuar este flagelo².

\section{CONCLUSÃO}

Através do estudo constatou-se que as principais causas de trauma abdominal foram ferimentos por arma de fogo (trauma aberto) e quedas (trauma fechado). As vísceras mais comumente atingidas foram as parenquimatosas (baço e fígado), no trauma fechado. Lesão de intestinos, fígado e rins, no trauma aberto. Dentre os pacientes atendidos, a maioria era do sexo masculino e a faixa etária predominante foi entre 21 e 30 anos. Nos politraumatizados, outras regiões foram afetadas além do abdômen, sendo a mais acometida o tórax.

Ribas-Filho JM, Malafaia O, Fouani MM, Justen MS, Pedri LE, Silva LMA, Mendes JF. Abdominal trauma: study of the most frequent wounds of digestive system and its causes. ABCD Arq Bras Cir Dig 2008;21(4):170-4

ABSTRACT - Background - Abdominal trauma is the distress resulting from a sudden and violent action effected by various agents. Its incidence has been increasing, and severity is determined by injury to vital structures in the abdomen and associated injuries. Aim - To identify the causes of abdominal trauma, the most frequently injured digestive viscera, the presence of injuries in other anatomic regions and the relationship of abdominal trauma to sex and age group. Method - Thirty-four patients from the Sistema Único de Saúde [the public healthcare system] were selected, all diagnosed with abdominal trauma and seen from January 2005 through September 2005 at the Hospital Universitário Evangélico de Curitiba. Data collection was performed with the aid of a previously formulated protocol. Results - It was found that $91 \%$ of the victims were males. The most affected age group was in its third decade of life. Regarding the classification of traumatic injuries, $58.82 \%$ presented with open trauma wounds and $41.18 \%$ with contusions. Falls accounted for $44 \%$ of contusions, followed by traffic accidents with $35 \%$. The most common open wounds were caused by firearms in $56 \%$ of cases and by knives in $44 \%$. Small intestine injury occurred in $31 \%$ of the open wounds, followed by liver, colon and kidney injury, with $23 \%$ each. In contusions, $60 \%$ of the patients sustained spleen injuries. The thorax was the region most frequently associated with abdominal trauma (31\%). Conclusion - The leading causes of abdominal trauma were gunshot wounds (penetrating trauma) and falls (blunt trauma). The most frequently injured viscera in blunt trauma were the parenchymatous ones (spleen and liver), and intestines, liver and kidneys in penetrating trauma. Most patients were males, predominantly in their third decade of life.

HEADINGS - Abdominal injuries. Contusions. Wounds and injuries. Firearms. Accidents, Traffic .

\section{REFERÊNCIAS}

1. Andrade SM, Jorge MHP. Acidentes de transporte terrestre em município da região sul do Brasil. Rev Saúde Pública 2001;35(3):318-20.

2. Batista JN, Gomes EGA. Etiologia do trauma. In: Freire E. Trauma: a doença dos séculos 2001;1(1):17-45.

3. Batista SEA, Baccani JG, Silva RAP, Gualda KPF, Vianna Jr., Raul JA. Análise comparativa entre os mecanismos de trauma, as lesões e o perfil de gravidade das vítimas, em Catanduva - SP. Rev Col Bras Cir. 2006;33(1):6-10.

4. Birolini D. Como anda a epidemia de trauma? Rev Assoc Med Bras. 2001;47(1):3.

5. Carvalho Neto JA. Mortalidade por violências e acidentes no Distrito Federal: a situação entre 1980 e 1994. Brasília Méd. 2000;37(3/4):99-108.

6. Croce MA, Fabian TC, Menke PG, Waddle-Smith L, Minard G, Kudsk KA, Patton JH Jr, Schurr MJ, Pritchard FE. Nonoperative management of blunt hepatic trauma is the treatment of choice for hemodynamically stable patients. Results of a prospective trial. Ann Surg. 1995 Jun;221(6):744-53.

7. Davis JJ, Cohn JrI, Nance FC. Diagnosis and management of blunt abdominal trauma. Ann Surg. 1976;185:672.

8. DiVincenti FC, Rives JD, Laborde EJ, Fleming ID, Cohn I Jr. Blunt abdominal trauma. J Trauma. 1968 Nov;8(6):1004-13.
9. Edwards J, Gaspard DJ. Visceral injury due to extraperitoneal gunshot wounds. Arch Surg. 1974 Jun;108(6):865-6.

10. Fagundes MAV, Seidel AC, Schiavon AC, Barbosa FS, Kanamaru F. Estudo retrospectivo de janeiro de 1998 a maio de 2005, no Hospital Universitário de Maringá, sobre ferimentos por arma branca e arma de fogo. Acta Sci Health Sci. 2007;29(2):133-7.

11. Hurtuk M, Reed RL 2nd, Esposito TJ, Davis KA, Luchette FA. Trauma surgeons practice what they preach: The NTDB story on solid organ injury management. J Trauma. 2006 Aug;61(2):243-54.

12. Kemmeter PR, Hoedema RE, Foote JA, Scholten DJ. Concomitant blunt enteric injuries with injuries of the liver and spleen: a dilemma for trauma surgeons. Am Surg. 2001 Mar;67(3):221-5.

13. Martins S, Souto MID. Emergências cirúrgicas traumáticas. Manual de Emergências Médicas: Diagnóstico e Tratamento. Rio de Janeiro, Revinter, 1998; 369:373.

14. Portela CAS, Fernández JCR, Arias LER, Rodríguez LS, Arteaga YD. Morbilidad y mortalidad por traumatismo abdominal. Rev Cubana Cir 2007; 46 (3). 
15. Prado Filho OR, Pazello DR, Colferal DR, Daniel MJ, Vasconcelos VMF. Caracterização dos traumas abdominais em pacientes atendidos no Hospital Universitário Regional de Maringá em 2006. Acta Sci Health Sci Maringá 2008; 30(2):129-32.

16. Rasslan S. Trauma Abdominal. Afecções Cirúrgica de Urgência 1995; 293:304.

17. Ribas Filho J, Malafaia O, Campos ACL, Grauman RQ, Gomes SE, Marochi VL. Prevalência das estruturas atingidas no Trauma Abdominal. Rev Méd Paraná. 2002;60(1):25-9.

18. Riveros A, Urbina I, Díaz L, Ramírez R, Calvo V, Duran L. Revisión de traumatismos abdominales en el Hospital Central de San Cristobal: Estado Táchira 1994-1998. Col Med Estado Táchira, 2003;12(3):27-31.

19. Hayt DB, Coimbra R, Potenza B. Tratamento do trauma agudo. In: Sabiston Jr DC. Tratado de cirurgia. $17^{\mathrm{a}}$ ed. Rio de Janeiro: Elsevier; 2005. v.1, p.512..

20. Sonneborn Gross R, Espinosa GR, Geni GR, Ricardo RW, Alejandra PG, Erik PRZM. Resultados del tratamiento de 588 pacientes con trauma múltiple. Rev Med Chile 1998;126(12):1478-82.
21. Stalhschmidt CMM, Formighieri B, Lubachevski FL. Controle de danos no trauma abdominal e lesões associadas: experiência de cinco anos em um serviço de emergência. Rev Col Bras Cir. 2006;33(4).215-9.

22. Stracieri LD, Scarpelini S. Trauma hepático. Acta Cir Bras. 2006;21(Supl.1):858.

23. Tambellini AT, Osanai CH. Epidemiologia do Trauma. In: Freire E, editor. Trauma: a doença dos séculos 2001; 47-76.

24. Wilson H, Sherman R. Civilian penetrating wounds of the abdomen. Ann. Surg;1961;153:639.

Fonte de financiamento: não há Conflito de interesse: não há Recebido para publicação: 01/06/2008 Aceito para publicação: 30/09/2008 